\title{
Association between glycemic control and antidiabetic drugs in type 2 diabetes mellitus patients with cardiovascular complications
}

This article was published in the following Dove Press journal:

Drug Design, Development and Therapy

18 August 2015

Number of times this article has been viewed

\author{
Hasniza Zaman Huri, ${ }^{1,2}$ \\ Doris Yew Hui Ling' \\ Wan Azman Wan Ahmad 2,3 \\ 'Department of Pharmacy, Faculty \\ of Medicine, University of Malaya, \\ Kuala Lumpur, Malaysia; ${ }^{2}$ Clinical \\ Investigation Centre, University \\ Malaya Medical Centre, Lembah \\ Pantai, Kuala Lumpur, Malaysia; \\ ${ }^{3}$ Cardiology Unit, Department \\ of Medicine, Faculty of Medicine, \\ University of Malaya, Kuala Lumpur, \\ Malaysia
}

Purpose: Cardiovascular disease (CVD) is a macrovascular complication in patients with type 2 diabetes mellitus (T2DM). To date, glycemic control profiles of antidiabetic drugs in cardiovascular (CV) complications have not been clearly elucidated. Therefore, this study was conducted retrospectively to assess the association of antidiabetic drugs and glycemic control with CV profiles in T2DM patients. The association of concurrent medications and comorbidities with glycemic control was also investigated.

Methods: A total of 220 T2DM patients from the University of Malaya Medical Centre, Malaysia, who had at least one CV complication and who had been taking at least one antidiabetic drug for at least 3 months, were included. The associations of antidiabetics, cardiovascular diseases, laboratory parameters, concurrent medications, comorbidities, demographics, and clinical characteristics with glycemic control were investigated.

Results: Sulfonylureas in combination $(P=0.002)$ and sulfonylurea monotherapy $(P<0.001)$ were found to be associated with good glycemic control, whereas insulin in combination ( $P=0.051)$, and combination biguanides and insulin therapy $(P=0.012)$ were found to be associated with poor glycemic control. Stroke $(P=0.044)$ was the only type of CVD that seemed to be significantly associated with good glycemic control. Other factors such as benign prostatic hyperplasia $(P=0.026)$, elderly patients $(P=0.018)$, low-density lipoprotein cholesterol levels $(P=0.021)$, and fasting plasma glucose $(P<0.001)$ were found to be significantly correlated with good glycemic control.

Conclusion: Individualized treatment in T2DM patients with CVDs can be supported through a better understanding of the association between glycemic control and CV profiles in T2DM patients.

Keywords: glycemic control, antidiabetic drugs, type 2 diabetes mellitus, cardiovascular disease

\section{Introduction}

Diabetes mellitus (DM) is a chronic disease and a growing epidemic that has become a global public health concern. ${ }^{1}$ Approximately 366 million people worldwide have $\mathrm{DM}$, and this number is expected to increase to 552 million adults by $2030 .^{2}$ According to the National Diabetes Information Clearing house and the World Health Organization, up to $90 \%$ of diabetics have type 2 diabetes mellitus (T2DM). The epidemiology of T2DM has risen from $20.8 \%$ in 2011 to $22.9 \%$ in 2013 among adults 30 years and older in Malaysia. ${ }^{3}$

T2DM is associated with chronic complications that are subdivided into microvascular and macrovascular complications. ${ }^{4}$ Cardiovascular disease (CVD), which
Correspondence: Hasniza Zaman Huri Department of Pharmacy, Faculty of Medicine, University of Malaya, 50603 Kuala Lumpur, Malaysia

Tel +60 379676659

Fax +60 379674964

Email hasnizazh@um.edu.my 
is a common macrovascular complication, is a major cause of mortality and morbidity in T2DM patients, representing approximately $50 \%$ of all fatalities. ${ }^{4}$ According to the World Heart Federation, people with diabetes are 2-4 times more likely to develop CVDs. ${ }^{5}$

Intensive glycemic control that targets a glycated hemoglobin $\left(\mathrm{A}_{1 \mathrm{C}}\right)$ of $7 \%$ and below can help to prevent or slow the progression of microvascular complications such as diabetic retinopathy and chronic kidney disease (CKD) in T2DM patients. ${ }^{4}$ In contrast, there is limited information on the association between glycemic control and macrovascular diseases such as CVDs. ${ }^{6}$ The glycemic control profiles of antidiabetic drugs in various cardiovascular complications have not been clearly elucidated.

Although the role of antidiabetic drugs in CVD progression in T2DM patients has not been determined, some studies have shown that some antidiabetic agents cause an improvement in CVD progression in these patients. For example, glucagon-like peptide-1 (GLP-1) receptor agonists can reduce $\mathrm{A}_{1 \mathrm{C}}$ by approximately $1 \%-1.5 \%$ via glucose-dependent secretion of insulin. ${ }^{7}$ Glucose-dependent secretion of insulin can then help to increase glucose use and intensify the metabolic control, which improves myocardial metabolism and myocardial function. ${ }^{8}$ A study showed that a 72-hour infusion with the GLP-1 receptor agonist in human subjects with acute myocardial infarction improved the left ventricular ejection fraction (LVEF). ${ }^{9}$ Sokos et al ${ }^{10}$ demonstrated that a 5-week infusion of GLP-1 receptor agonist in both diabetic and chronic heart failure (HF) patients (New York Heart Association [NYHA] class III and IV) significantly improved LVEF in patients with HF.

Metformin is a first-line oral antidiabetic drug used in type 2 diabetes therapy that decreases hepatic glucose production and increases glucose utilization. ${ }^{11}$ Shah et $\mathrm{al}^{12}$ found that glycemic control with metformin significantly improved the LVEF in patients with advanced systolic HF (NYHA class III and IV) during a 6-month follow-up. Soraya et $\mathrm{al}^{13}$ showed that metformin $(25 \mathrm{mg} / \mathrm{kg})$ lowered left ventricular end-diastolic pressure in rats with isoproterenol-induced myocardial infarction. Thus, antidiabetic drugs can be used to improve the clinical parameters of various cardiovascular (CV) complications in T2DM patients.

This study aims to: 1) assess the association between glycemic control, antidiabetic drugs and ischemic heart disease (IHD), acute coronary syndrome (ACS), HF, atrial fibrillation (AF), and/or stroke in T2DM patients; 2) study the association of glycemic control and comorbidities that presented in T2DM patients with IHD, ACS, HF, AF, and/or stroke; and
3) determine the association between glycemic control and concomitant medications taken by T2DM patients with IHD, ACS, HF, AF, and/or stroke. Data obtained from this study may be used to improve the $\mathrm{CV}$ profile in T2DM patients by improving glycemic control and the use of antidiabetic agents. The results could enhance our understanding of the association between glycemic control, antidiabetic agents and the profile of CV complications in T2DM patients. This study promotes and supports the concept of pharmaceutical care and individualized treatment in T2DM patients with CV complications.

\section{Material and methods Study design and setting}

This retrospective study was conducted at the University of Malaya Medical Centre (UMMC), Malaysia, a premier teaching hospital in Malaysia with 1,200 beds. The study was conducted in accordance with the Declaration of Helsinki and was approved by the medical ethics committee of the UMMC (reference number 1031.38). The medical ethics committee of the UMMC waived the need for written informed consent from the participants.

\section{Sample population, size, and time frame}

The population of this study consisted of T2DM patients at UMMC, who had CV complications and who had been taking at least one antidiabetic drug for at least 3 months.

The sampling time frame for this study was 5 years, from January 1, 2009 to March 31, 2014. The minimum sample size for this study was calculated using the Epi Info Program Version 7.0 (CDC, Atlanta, GA, USA). The level of significance, $\alpha$, was set at 0.05 , and the desired power of the study, $1-\beta$, was $80 \%$. Assuming that the expected proportion of T2DM patients on antidiabetic medications was $22.9 \%$ and the confidence limit was $5 \%$, the minimum sample size was 116 patients.

\section{Study population and procedures}

The potential patient population included a total of 1,167 patients whose registration numbers were identified using the Hospital Information System, based on the International Statistical Classification of Diseases Tenth Revision (ICD-10) for T2DM (E 11.0-E 11.8). A total of 220 patients fulfilled the inclusion criteria, as shown in Table 1, and they were included in the study.

Data collection forms were used to record patient data. Types of CV complications included in the study were IHD, ACS, HF, AF, and stroke. Patient demographic information 
Table I Inclusion and exclusion criteria of the study

\section{Inclusion criteria}

I) Patients who were diagnosed with T2DM.

2) Patients who were diagnosed with at least one cardiovascular complication (IHD, ACS, HF, AF, and/or stroke).

3) Patients who were prescribed at least one oral antidiabetic agent and/or insulin.

4) Patients who received antidiabetic therapy for at least 3 months.

Exclusion criteria

I) Patients who received nonpharmacological therapy/diet.

2) Patients who were below 18 years old.

Abbreviations:T2DM,type2diabetesmellitus; $\mathrm{HD}$, ischemicheartdisease; $\mathrm{ACS}$, acute coronary syndrome; $\mathrm{HF}$, heart failure; $\mathrm{AF}$, atrial fibrillation.

such as age, sex, ethnicity, height, weight, and body mass index (BMI) was collected. Other patient data such as comorbidity, duration of diabetes, social history (such as smoking and alcohol consumption), laboratory results, medication regimen, and concurrent medications were also collected and reviewed (Table 2).

\section{Statistical analysis}

All statistical analyses were performed using the statistical software Statistical Package for Social Science (SPSS), version 20 (IBM SPSS Inc., Chicago, IL, USA). Descriptive statistics such as frequencies, percentages, means, ranges and standard deviations (SD) were used to describe the data. Categorical data were expressed as the absolute number and percentage, whereas continuous data were expressed as the mean \pm SD if normally distributed or as the median (interquartile range) if skewed. The Kolmogrov-Smirnov test or the Shapiro-Wilk test was used to test the normality of continuous data. The Pearson chi-square test with continuity correction was used to examine the association between categorical variables. When the expected cell count was more than $20 \%$ or less than 5 , the Fisher exact test was used. Cramer's $V$ correlation test was used to examine the correlation between two nominal groups, such as antidiabetic regimens and $\mathrm{A}_{1 \mathrm{C}}$. The Pearson correlation was used to examine the correlation between two continuous data groups, such as low-density lipid and $\mathrm{A}_{1 \mathrm{C}}$. The significance level was accepted at $P<0.05$. The summarized findings are presented in Figure 1.

\section{Results Demographic characteristics}

A total of 1,167 T2DM patients fulfilled the requirement of ICD-10 (E 11.0-E 11.8) from January 1, 2009 to March 31, 2014, and 988 patients were selected through convenient sampling. Of the 520 patients whose medical records were successfully retrieved, 220 fulfilled the inclusion criteria. The study population consisted of slightly more ( $8.2 \%)$ male than female patients. The largest ethnic population in this study was Indian, followed by Malay, Chinese, and others.

As shown in Table 3, the patients' age was normally distributed when tested using the Kolmogrov-Smirnov test, with a mean \pm SD age of $64.7 \pm 11.4$ years (range, 36-91 years). There were more nonelderly patients $(54.1 \%)$ than elderly patients $(49.5 \%)$ in the study.

BMI was available for 94 patients (47.3\%). The BMI was normally distributed, with a mean \pm SD of $26.5 \pm 4.7 \mathrm{~kg} / \mathrm{m}^{2}$ (range, $18.20-38.97 \mathrm{~kg} / \mathrm{m}^{2}$ ). Most of the study subjects were obese, followed by preobese, normal range, and underweight.

\section{Clinical characteristics}

Most patients with CV complications had had T2DM for more than 20 years (Table 4 ). A total of $65.9 \%$ of patients were nonsmokers. IHD had the highest frequency of CVD types. This was followed by ACS, stroke, HF, and AF. Non-ST-elevation myocardial infarction was the most common type of ACS, followed by unstable angina and ST-elevation myocardial infarction. The NYHA class IV was the most common type of HF, followed by NYHA III, II, and I.

\section{Laboratory parameters}

LVEF data were available for 125 patients (Table 5). The data were normally distributed and the mean LVEF was $49.0 \% \pm 19.7 \%$ (range, $10.0 \%-85.0 \%$ ).

Table 2 Definitions used in the study

\begin{tabular}{ll}
\hline Terms & Definition \\
\hline $\begin{array}{l}\text { Cardiovascular } \\
\text { complications }\end{array}$ & $\begin{array}{l}\text { One of the macrovascular complications that occurred in T2DM patients that involves heart and blood vessels, such } \\
\text { as coronary heart disease, cerebrovascular disease, peripheral arterial disease, rheumatic heart disease, congenital } \\
\text { heart disease as well as deep vein thrombosis and pulmonary embolism. }{ }^{4}\end{array}$ \\
$\begin{array}{l}\text { Comorbidities } \\
\text { Good glycemic control }\end{array}$ & Diseases or other pathological processes that occur simultaneously with another and require long-term treatment. ${ }^{42}$ \\
Poor glycemic control & Targeted $\mathrm{A}_{\mathrm{IC}}$ levels below $8 \%$ within 3 months. ${ }^{4}$ \\
\hline
\end{tabular}

Abbreviations: T2DM, type 2 diabetes mellitus; $A_{I C}$, glycated hemoglobin. 
Approved by the medical ethics committee of the UMMC

(reference number: 1031.38)

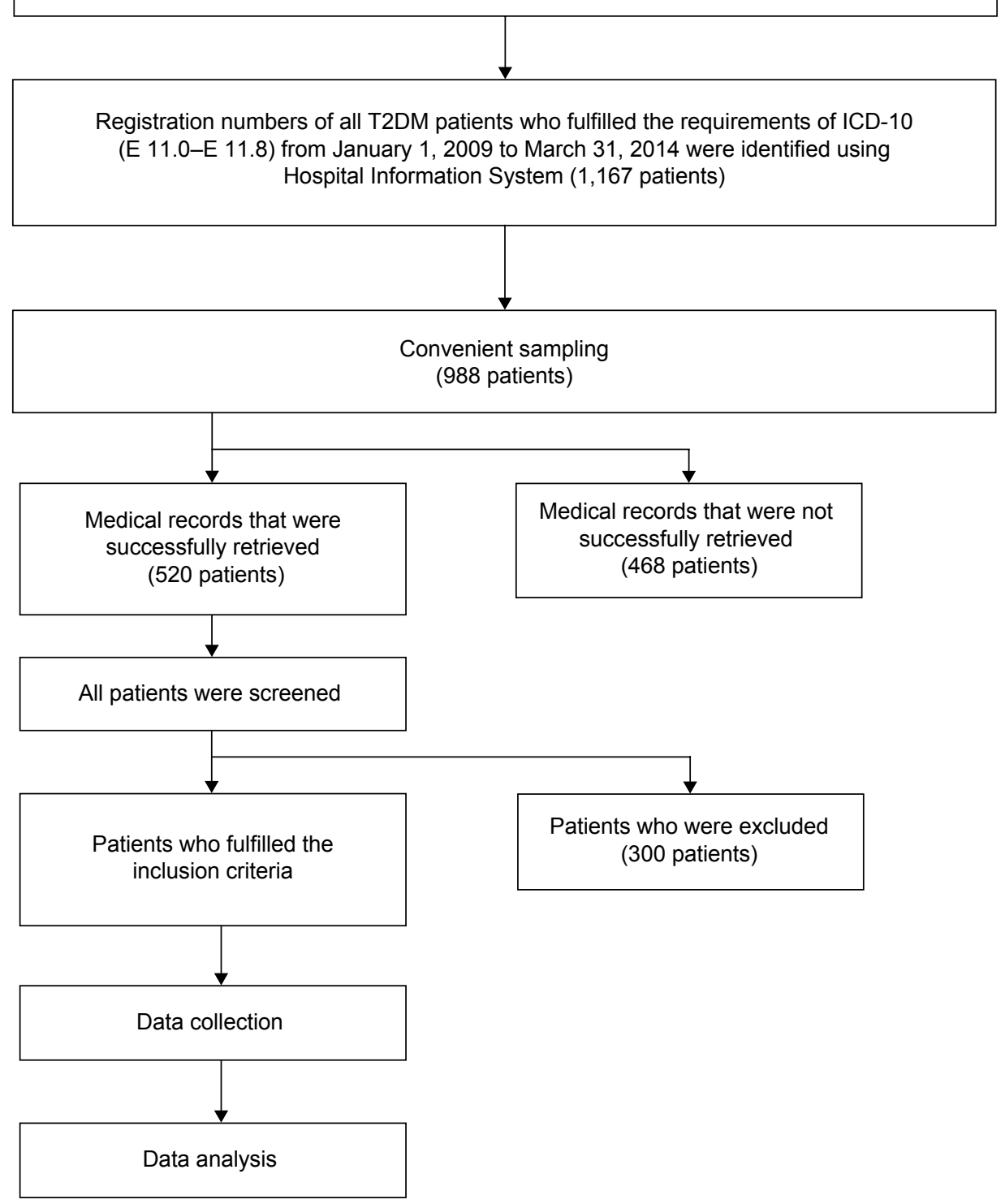

Figure I Overview of methodology.

Abbreviations: UMMC, University of Malaya Medical Centre; T2DM, type 2 diabetes mellitus; ICD-I0, International Statistical Classification of Diseases Tenth Revision.

Overall, the majority $(56.8 \%)$ of patients had achieved the target glycemic control $\left(\mathrm{A}_{1 \mathrm{C}}<8.0 \%\right)$. $\mathrm{A}_{1 \mathrm{C}}$ was normally distributed, and the mean $\mathrm{A}_{1 \mathrm{C}}$ was $8.1 \% \pm 2.0 \%$.

\section{Antidiabetic regimens}

Figure 2 shows the patients' antidiabetic regimens. Insulin monotherapy was the most commonly used antidiabetic regimen in this study, followed by sulfonylurea monotherapy, and biguanide and sulfonylurea combination therapy.

Figure 3 shows the patients' biguanide regimen. Metformin $500 \mathrm{mg}$ twice daily was the most common dosing regimen in the biguanide-treated group. This was followed by metformin $850 \mathrm{mg}$ extended-release twice daily and metformin $850 / 1,700 \mathrm{mg}$ twice daily.

Figure 4 shows the patients' sulfonylurea regimen. For the sulfonylurea-treated group, gliclazide $60 \mathrm{mg}$ modifiedrelease (MR) once daily was used most frequently. This was followed by gliclazide $30 \mathrm{mg}$ MR once daily and gliclazide $120 \mathrm{mg}$ MR once daily.

Figure 5 shows the patients' insulin regimen. For the insulin-treated group, most patients were taking a combination of isophane insulin and soluble insulin subcutaneously. 
Table 3 Demographic characteristics of patients $(N=220)$

\begin{tabular}{|c|c|}
\hline $\begin{array}{l}\text { Demographic characteristics } \\
(\mathrm{N}=\mathbf{2 2 0})\end{array}$ & $\begin{array}{l}\text { Number } \\
\text { (percentage, \%) }\end{array}$ \\
\hline \multicolumn{2}{|l|}{ Sex } \\
\hline Male & $119(54.1)$ \\
\hline Female & $101(45.9)$ \\
\hline \multicolumn{2}{|l|}{ Age } \\
\hline Nonelderly & III (50.5) \\
\hline Elderly & $109(49.5)$ \\
\hline \multicolumn{2}{|l|}{ Ethnicity } \\
\hline Malay & $81(36.8)$ \\
\hline Chinese & $55(25.0)$ \\
\hline Indian & $83(37.7)$ \\
\hline Other & $\mathrm{I}(0.5)$ \\
\hline \multicolumn{2}{|l|}{ Body mass index (BMI) } \\
\hline Underweight $(<18.5)$ & $2(0.9)$ \\
\hline Normal range (18.5-22.9) & $25(11.4)$ \\
\hline Preobese (23.0-27.4) & $34(15.5)$ \\
\hline Obese $(\geq 27.5)$ & $42(19.1)$ \\
\hline Unknown & $117(53.2)$ \\
\hline
\end{tabular}

Note: BMI classification is based on Clinical Practice Guidelines on Management of Obesity. ${ }^{43}$

Table 4 Clinical characteristic of patients $(N=220)$

\begin{tabular}{|c|c|}
\hline $\begin{array}{l}\text { Clinical characteristics } \\
(\mathrm{N}=220)\end{array}$ & $\begin{array}{l}\text { Number } \\
\text { (percentage, \%) }\end{array}$ \\
\hline \multicolumn{2}{|l|}{ Duration of T2DM (years) } \\
\hline$<1$ & $3(1.4)$ \\
\hline $1-5$ & $17(7.7)$ \\
\hline $6-10$ & $36(16.4)$ \\
\hline $11-15$ & $29(13.2)$ \\
\hline $16-20$ & $18(8.2)$ \\
\hline$>20$ & $53(24.1)$ \\
\hline Unknown & $64(29.1)$ \\
\hline \multicolumn{2}{|l|}{ Smoking status } \\
\hline Yes & $25(11.4)$ \\
\hline No & 145 (65.9) \\
\hline Ex-smoker & $38(17.3)$ \\
\hline Unknown & $12(5.5)$ \\
\hline \multicolumn{2}{|l|}{ Types of CVDs ${ }^{\mathrm{a}}$} \\
\hline IHD & $172(78.2)$ \\
\hline ACS & $115(52.3)$ \\
\hline NSTEMI & $60(52.7)$ \\
\hline STEMI & $18(15.5)$ \\
\hline UA & $38(32.8)$ \\
\hline $\mathrm{HF}$ & $29(13.2)$ \\
\hline NYHA I & $5(16.7)$ \\
\hline NYHA II & $6(20.0)$ \\
\hline NYHA III & $8(26.7)$ \\
\hline NYHA IV & II (36.7) \\
\hline $\mathrm{AF}$ & $12(5.5)$ \\
\hline Stroke & $49(22.3)$ \\
\hline
\end{tabular}

Note: ${ }^{2}$ Each patient may have more than one type of CVD.

Abbreviations: CVDs, cardiovascular diseases; IHD, ischemic heart disease; ACS, acute coronary syndrome; NSTEMI, non-ST-elevation myocardial infarction; STEMI, ST-elevation myocardial infarction; UA, unstable angina; $\mathrm{HF}$, heart failure; NYHA, New York Heart Association; AF, atrial fibrillation;T2DM, type 2 diabetes mellitus.
Table 5 Laboratory parameters

\begin{tabular}{lll}
\hline Variables & $\mathbf{N}$ & Values \\
\hline LVEF $(\%)$ & & \\
$\quad$ Increased LVEF $(>75 \%)$ & 220 & $13(5.9)$ \\
$\quad$ Normal LVEF (50\%-75\%) & & $53(24.1)$ \\
$\quad$ Moderately reduced (30\%-49\%) & & $34(15.5)$ \\
$\quad$ Severely reduced (<30\%) & & $24(10.9)$ \\
$\quad$ Unknown & & $96(43.6)$ \\
A IC $(\%)$ & & $25(56.8)$ \\
$<8.0 \%$ & 220 & $95(43.2)$ \\
$\geq 8.0 \%$ & & $10.2(5.0)$ \\
FBG (mmol/L) & 195 & $4.4(1.3)$ \\
Lipid profile & & $1.0(0.8-1.3)$ \\
Total cholesterol (mmol/L) & 211 & $2.4(1.8-3.1)$ \\
HDL (mmol/L) & 211 & $1.6(1.8-3.1)$ \\
LDL (mmol/L) & 210 & \\
Triglyceride (mmol/L) & 210 & $144(25.3)$ \\
Blood pressure (BP) & & $74(66.8-84.0)$ \\
SBP (mmHg) & 142 & \\
DBP (mmHg) & 142 & $121.0(76.0-243.0)$ \\
Cardiac biomarker & & $2.0(1.0-4.0)$ \\
CK (U/L) & 199 & $0.1(0.0-0.9)$ \\
CK-MB (U/L) & 196 & \\
Tnl (ng/L) & 154 &
\end{tabular}

Notes: Values presented as mean (SD) if normally distributed or median (interquartile range) if skewed for continuous data; $\mathrm{n}(\%)$ for categorical data.

Abbreviations: LVEF, left ventricular ejection fraction; $A_{I C}$, glycated hemoglobin; FBG, fasting blood glucose; HDL, high-density lipoprotein; LDL, low-density lipoprotein; SBP, systolic blood pressure; DBP, diastolic blood pressure; CK, creatine kinase; CK-MB, creatine kinase-MB; Tnl, Troponin-l.

This was followed by a subcutaneous (S/C) mixtard and S/C isophane insulin alone.

Figure 6 shows the patients' dipeptidyl peptidase-4 (DPP-4) inhibitor regimen. Sitagliptin $50 \mathrm{mg}$ and Vildagliptin $50 \mathrm{mg}$ were the two most commonly used DPP-4 inhibitors in the DPP-4 inhibitor-treated group.

Figure 7 shows the other antidiabetic agents with their dosing regimens. In this study, only two patients received acarbose $50 \mathrm{mg}$, one patient received acarbose $100 \mathrm{mg}$ ( $\alpha$-glucosidase inhibitor), one patient received exanatide $10 \mu \mathrm{g} / 40 \mu \mathrm{L}$ (glucose-like peptide-1 agonist), and one patient received rosiglitazone $4 \mathrm{mg}$ (thiazolidinedione).

\section{Comorbidities}

Figure 8 illustrates the common comorbidities in T2DM patients with CV complications. All patients with comorbidities had multiple comorbidities. Hypertension accounted for the highest percentage among all categories of comorbidities. This was followed by renal disease, hyperlipidemia, obesity, and retinopathy. Figure 9 shows that most patients with renal disease had CKD stage $3(30-59 \mathrm{~mL} / \mathrm{min})$, followed by CKD stages 4 and 5. Only five patients (2.3\%) had pneumonia, and ten $(4.5 \%)$ had hepatic disease. Of the five patients with pneumonia, three had hospital-acquired pneumonia, and two had 


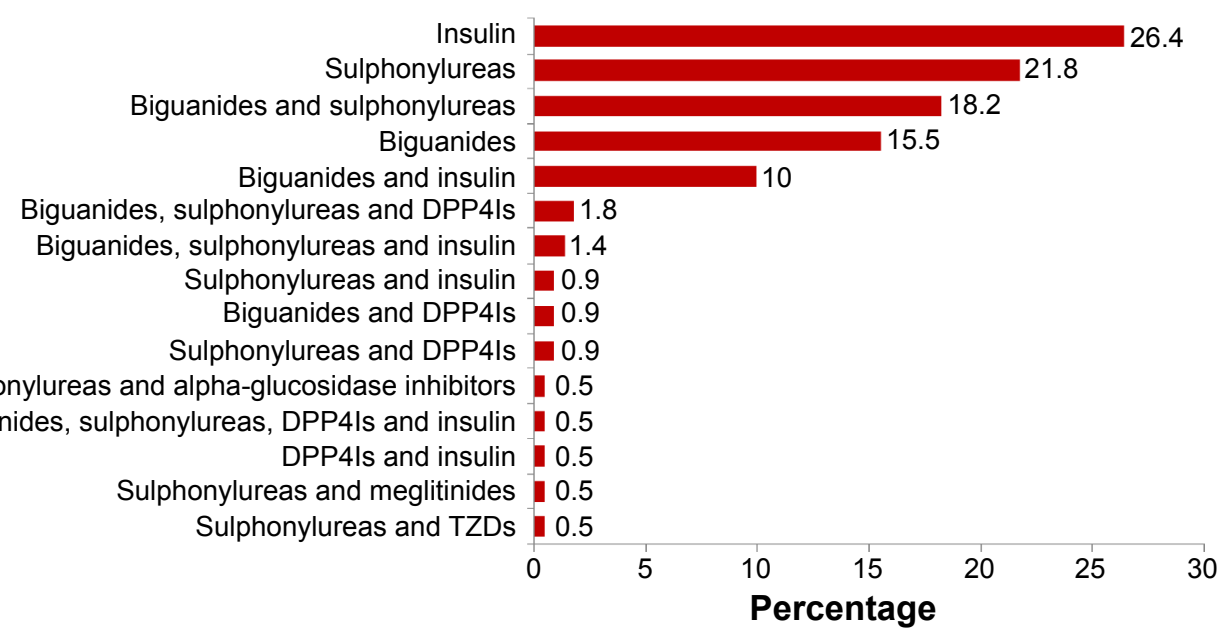

Figure 2 Antidiabetic regimens

Abbreviations: DPP4ls, dipeptidyl peptidase-4 inhibitors; TZDs, thiazolidinediones.

community-acquired pneumonia infections. Fatty liver was the most common hepatic disease, followed by hepatitis and cirrhosis (Figure 10). Other comorbidities included dementia (four cases), hypothyroidism (three), eye cataract (three), urinary tract infection (two), breast cancer (two), chronic obstructive pulmonary disease (one), gastritis (one), cholelithiasis (one), recurrent venous thrombosis (one), peptic ulcer disease (one), pulmonary tuberculosis (one), and osteoporosis (one). Only eleven patients $(5 \%)$ were without any comorbidity.

\section{Concurrent medications}

The pattern of concurrent medication use in T2DM patients with CV complications is summarized in Figure 11. Most patients had received antiplatelet therapy and statins as concurrent medications. Other concurrent medications included lactulose (two cases), bisacodyl (two), phenytoin (one), morphine (one), L-thyroxine (one), Renifer (vitamin B9 $1.5 \mathrm{mg}$, zinc sulfate $61.8 \mathrm{mg}$ and carbonyl iron $100 \mathrm{mg}$ ) (one), phenytoin (one), etoricoxib (one), Berodual metered-dose inhaler (one), Budesonide metered-dose inhaler (one), methyldopa (one), unasyn (one), piriton (one), cetirizine (one), motilim (one), duodart (dutasteride and tamsulosin $\mathrm{HCl}$ ) (one), and gabapentin (one). Supplements included multivitamins, calcitriol, calcium bicarbonate, vitamin B complex, and Ketosteril.

\section{Association between antidiabetic regimens and glycemic control}

There was a strong correlation between antidiabetic regimens and the $\mathrm{A}_{1 \mathrm{C}}$ level $\left(\chi^{2}=0.33, P=0.002\right)$. There was a significant association between glycemic control and insulin use

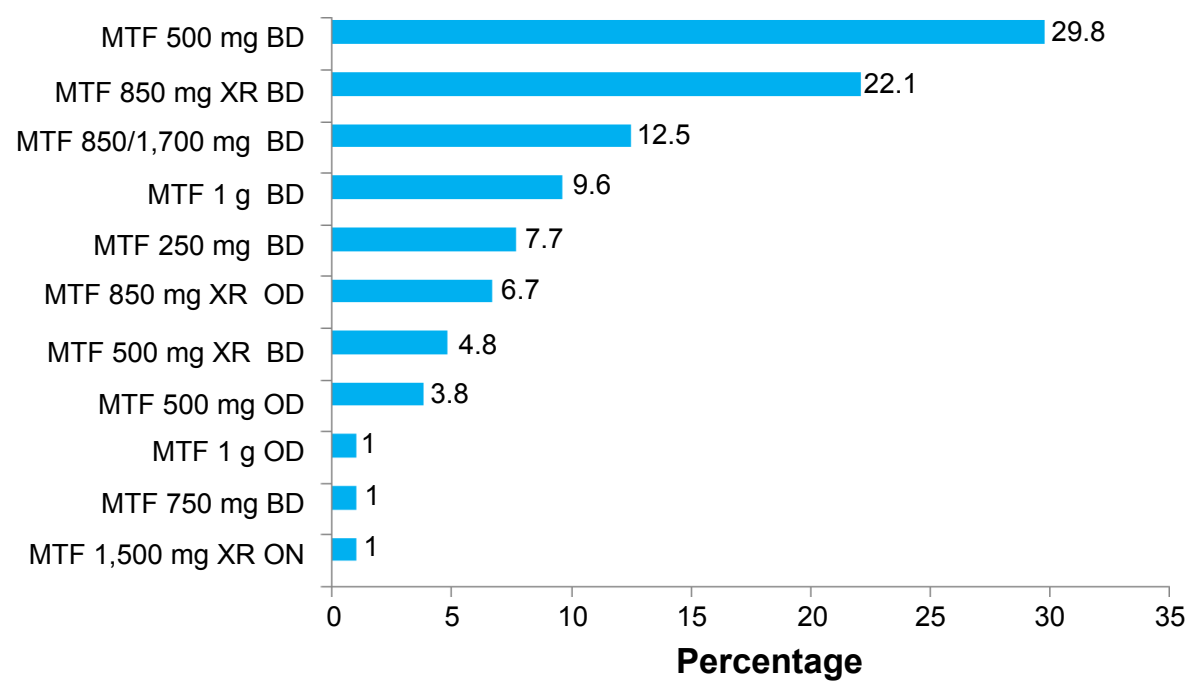

Figure 3 Dosing regimen of biguanides.

Abbreviations: MTF, metformin; XR, extended release; OD, once daily; BD, twice daily; ON, every night. 


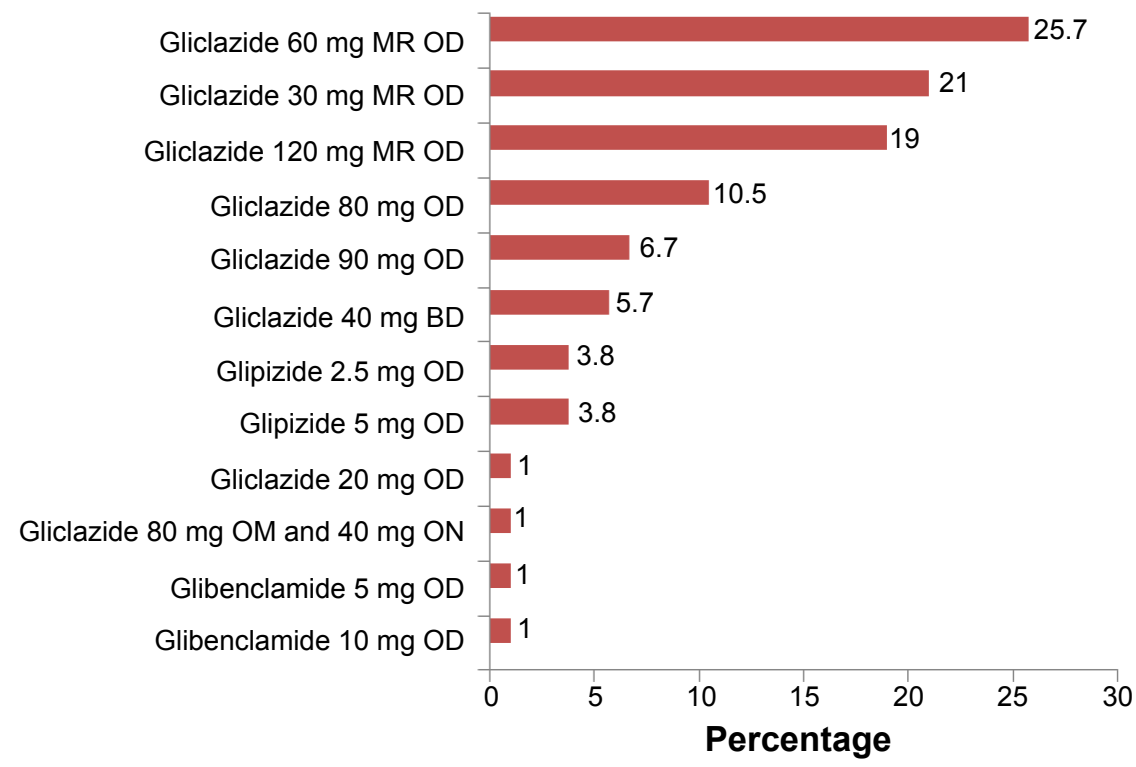

Figure 4 Dosing regimen of sulfonylureas.

Abbreviations: $\mathrm{OD}$, once daily; $\mathrm{BD}$, twice daily; MR, modified release; $\mathrm{ON}$, every night.

$\left(\chi^{2}=20.4, d f=1, P<0.001\right)$ as well as combination sulfonylurea therapy ( $\chi^{2}=9.4, d f=1, P=0.002$; Table 6 ). In addition, sulfonylurea monotherapy $\left(\chi^{2}=14.173, d f=1, P<0.001\right)$ and the combination of biguanides and insulin were significantly associated with glycemic control $\left(\chi^{2}=9.8, d f=1, P=0.006\right)$. In this study, sulfonylureas were associated with good glycemic control, whereas insulin was associated with poor glycemic control. The combination of biguanides and insulin was also associated with poor glycemic control.

\section{Association between cardiovascular diseases and glycemic control}

The association between CVDs and glycemic control was explored (Table 7). Stroke was the only type of CVD found to be associated with glycemic control $\left(\chi^{2}=4.060, d f=1\right.$, $P=0.044)$. A higher proportion of patients with stroke had good glycemic control.

\section{Association between laboratory parameters and glycemic control}

Table 8 shows the correlation between laboratory parameters and glycemic control. There was a significant correlation between low-density lipoprotein (LDL) and glycemic control $\left(r^{2}=0.159, P=0.021\right)$. The correlation coefficient $(r)$ was +0.159 , which indicates a positive, but weak, correlation. Fasting blood glucose was significantly correlated with glycemic control $\left(r^{2}=0.418, P<0.001\right)$. The correlation coefficient $(r)$ was +0.418 , which showed a positive, but weak, correlation.

$\mathrm{S} / \mathrm{C}$ isophane insulin $300 \mathrm{iu} / 3 \mathrm{~mL}$ and $\mathrm{S} / \mathrm{C}$ soluble insulin $300 \mathrm{iu} / 3 \mathrm{~mL}$

$\mathrm{S} / \mathrm{C}$ mixtard

$\mathrm{S} / \mathrm{C}$ isophane insulin $300 \mathrm{iu} / 3 \mathrm{~mL}$

$\mathrm{S} / \mathrm{C}$ soluble insulin $300 \mathrm{iu} / 3 \mathrm{~mL}$ and $\mathrm{S} / \mathrm{C}$ lanctus

$\mathrm{S} / \mathrm{C}$ soluble insulin $300 \mathrm{iu} / 3 \mathrm{~mL} \quad 3.4$

$\mathrm{S} / \mathrm{C}$ lanctus 3.4

$\mathrm{S} / \mathrm{C}$ insulin glargine $300 \mathrm{iu} / \mathrm{mL}$ | 1.1

$\mathrm{S} / \mathrm{C}$ novomix | 1.1

S/C humalog and lanctus | 1.1

$\mathrm{S} / \mathrm{C}$ lanctus and novorapid | 1.1

\begin{tabular}{ccccc}
\hline 0 & 20 & 40 & 60 & 80 \\
Percentage
\end{tabular}

Figure 5 Dosing regimen of insulin. Abbreviation: S/C, subcutaneous. 


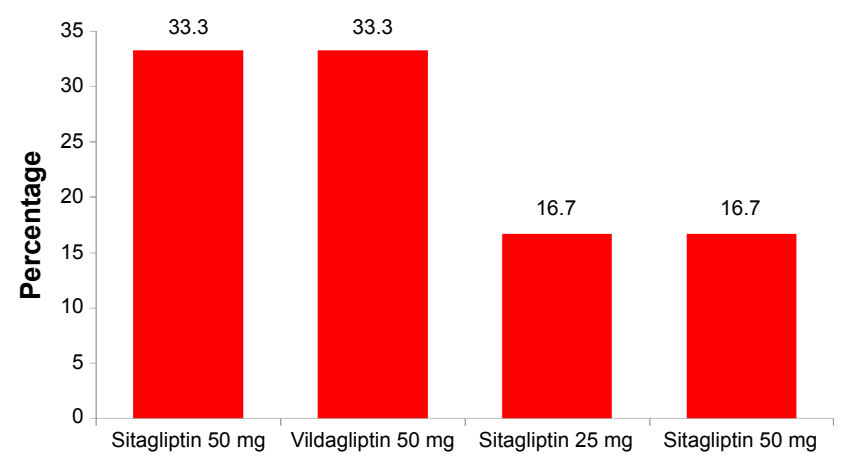

Figure 6 Dosing regimen of DPP-4 inhibitors.

Abbreviation: DPP-4 inhibitors, dipeptidyl peptidase-4 inhibitors.

\section{Association between concurrent medications and glycemic control}

The association between concurrent medications and glycemic control $\left(\mathrm{A}_{1 \mathrm{C}}\right)$ in T2DM patients with CVDs is presented in Table 9. No concurrent class of drugs was significantly associated with glycemic control.

\section{Association between comorbidities and glycemic control}

Benign prostatic hyperplasia (BPH) $\left(\chi^{2}=5.485, d f=1\right.$, $P=0.026$ ) was the only type of comorbidity that appeared to be significantly associated with glycemic control (Table 10). A higher proportion of patients with BPH had good glycemic control in this study compared to patients who did not obtain good glycemic control.

\section{Association of demographic and clinical characteristics with glycemic control}

The association between demographic and clinical characteristics and glycemic control is presented in Table 11. Age $\left(\chi^{2}=6.249, d f=1, P=0.018\right)$ was found to be significantly associated with glycemic control. In this study, the elderly group was more likely to have good glycemic control compared with the nonelderly group.

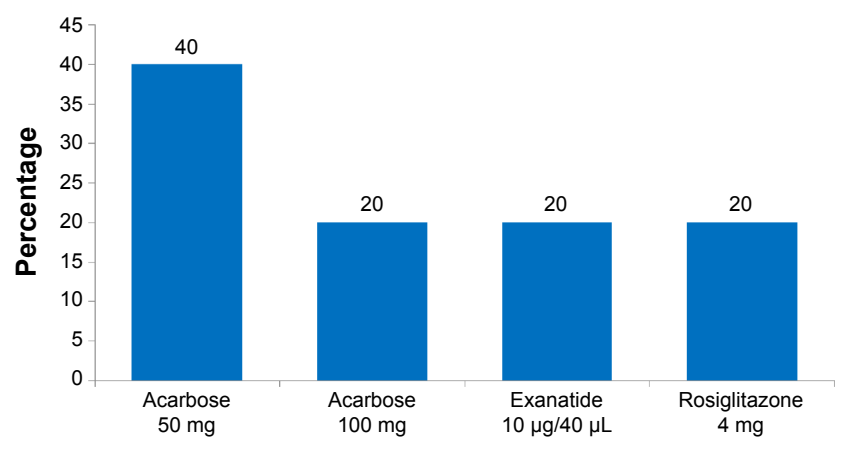

Figure 7 Dosing regimen of other antidiabetic regimens.

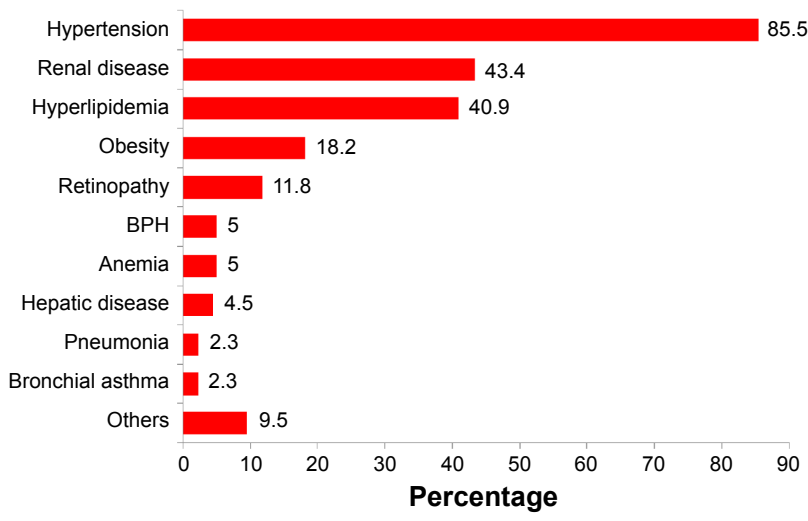

Figure 8 Comorbidities in T2DM patients with CVDs.

Note: Each patient may have more than one comorbidity.

Abbreviations: BPH, benign prostate hyperplasia; CVD, cardiovascular disease; T2DM, type 2 diabetes mellitus.

\section{Discussion}

\section{Association between antidiabetic agents and glycemic control}

\section{Sulfonylureas}

This study found that sulfonylurea use was significantly associated with good glycemic control. Sulfonylureas were the most commonly used antidiabetic agent in this study population; $\mathrm{A}_{1 \mathrm{C}}$ was reduced in these patients by $0.8 \%-2 \%$, and fasting plasma glucose (FPG) levels were reduced by $60-70 \mathrm{mg} / \mathrm{dL}$. ${ }^{7}$ Sulfonylureas stimulate the release of insulin from $\beta$-cells by binding to a specific sulfonylurea receptor on pancreatic $\beta$-cells. ${ }^{11}$

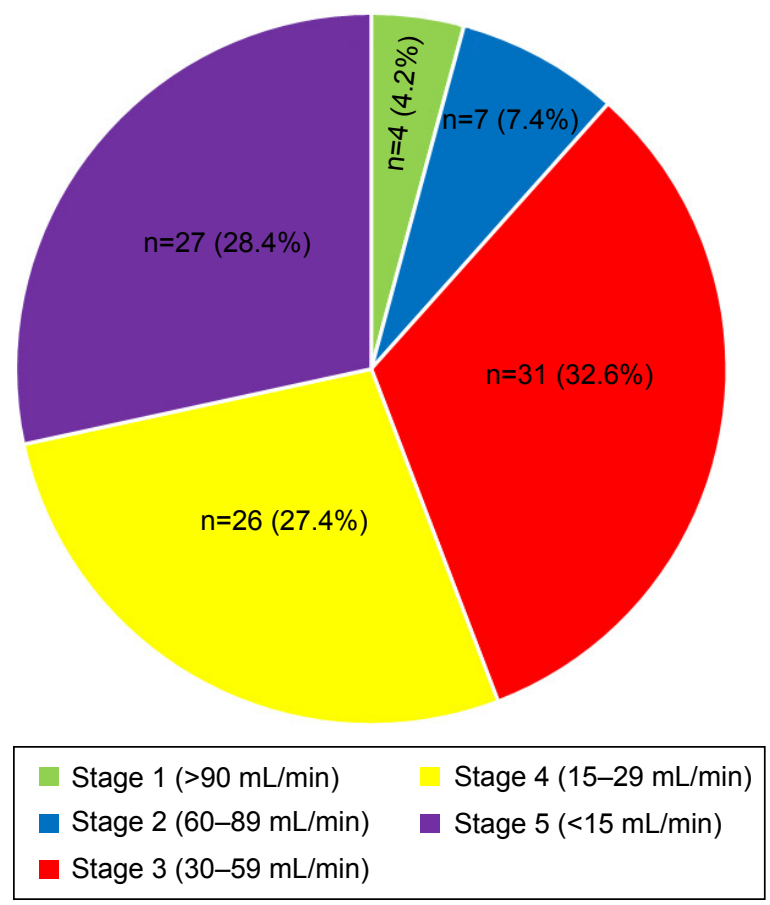

Figure 9 Stage of chronic kidney disease. 


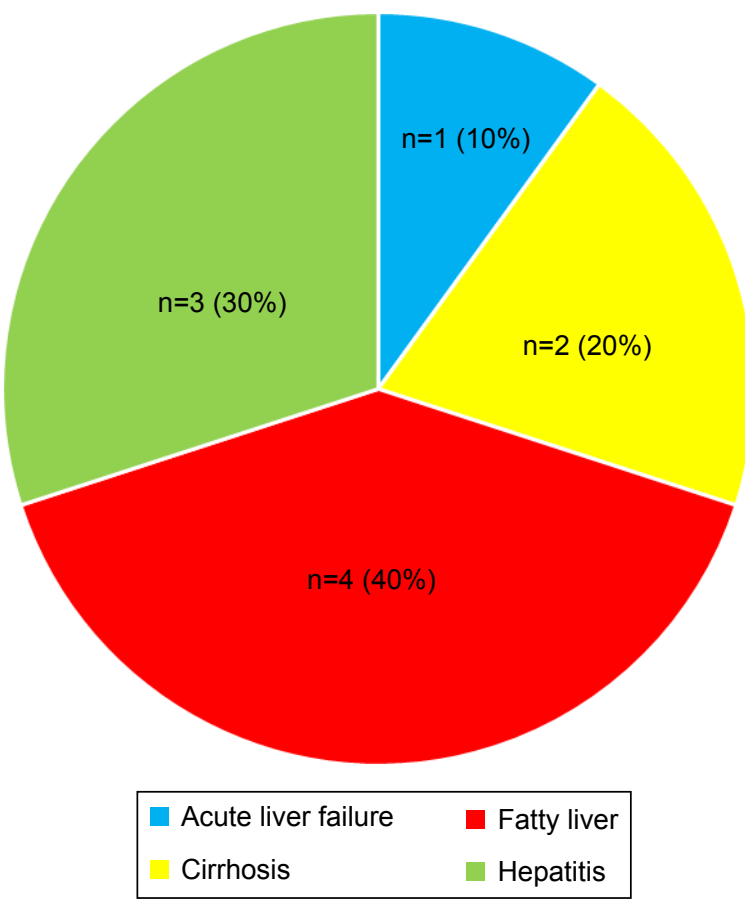

Figure 10 Types of liver disease.

In this study, gliclazide modified-release (MR) was the most common drug taken in the sulfonylurea-treated group. A study by Vilar et $\mathrm{al}^{14}$ showed that gliclazide MR monotherapy significantly improved glycemic control, with a $0.9 \%-1.8 \%$ reduction in $\mathrm{A}_{1 \mathrm{C}}$. In addition, gliclazide MR was associated with a significantly lower rate of hypoglycemia compared with glimepiride. ${ }^{15}$ In the sulfonylurea-treated group in this study, more patients were taking glipizide than

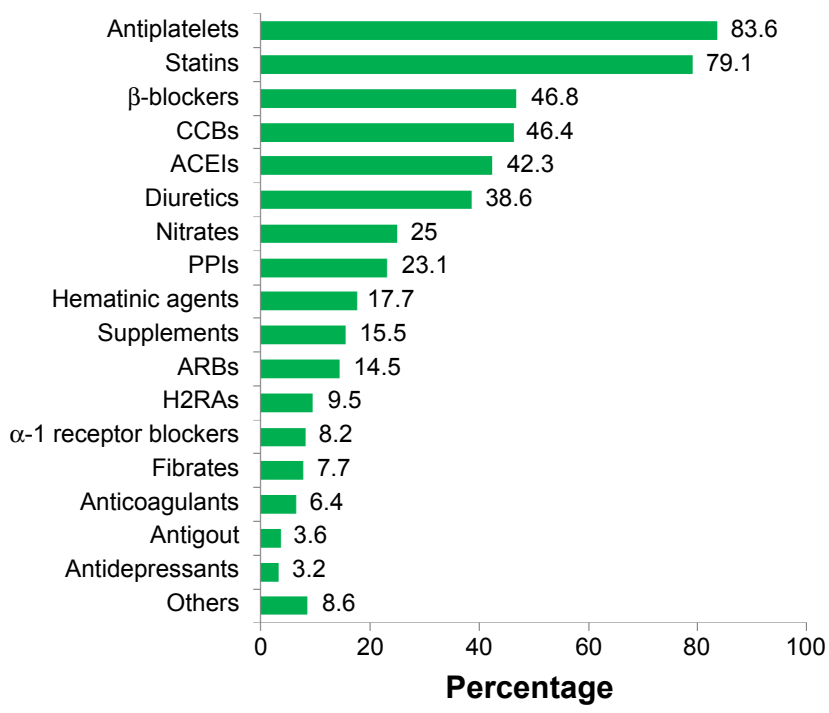

Figure I I Concurrent medications in T2DM patients with cardiovascular disease. Note: Each patient may take more than one concurrent medication.

Abbreviations: H2RAs, histamine 2 receptor antagonists; PPls, proton pump inhibitors; ACEls, angiotensin converting enzyme inhibitors; ARBs, angiotensin receptor blockers; T2DM, type 2 diabetes mellitus; CCBs, calcium channel blockers.
Table 6 Association between antidiabetic regimens and glycemic control

\begin{tabular}{|c|c|c|c|}
\hline \multirow{2}{*}{$\begin{array}{l}\text { Antidiabetic } \\
\text { regimens }\end{array}$} & \multicolumn{2}{|c|}{$A_{I C}$ level $(n, \%)$} & \multirow[t]{2}{*}{$P$-value ${ }^{a}$} \\
\hline & $<\mathbf{8 . 0} \%$ & $\geq \mathbf{8 . 0} \%$ & \\
\hline \multicolumn{4}{|l|}{ Biguanides } \\
\hline Yes & $52(49.5)$ & $53(50.5)$ & \multirow[t]{2}{*}{$P=0.05 \mathrm{I}^{\mathrm{b}}$} \\
\hline No & $73(63.5)$ & $42(36.5)$ & \\
\hline \multicolumn{4}{|c|}{ Sulfonylureas (in combination) } \\
\hline Yes & $72(60.2)$ & $34(32.1)$ & \multirow[t]{2}{*}{$P=0.002^{b, *}$} \\
\hline No & $53(46.5)$ & $61(53.5)$ & \\
\hline \multicolumn{4}{|l|}{ Meglitinides } \\
\hline Yes & I (I00.0) & $0(0.0)$ & \multirow[t]{2}{*}{$P=1.000^{c}$} \\
\hline No & $124(56.6)$ & $95(43.4)$ & \\
\hline \multicolumn{4}{|l|}{ TZDs } \\
\hline Yes & $0(0.0)$ & I (I00.0) & \multirow[t]{2}{*}{$P=0.432^{c}$} \\
\hline No & $125(57.1)$ & $94(42.9)$ & \\
\hline \multicolumn{4}{|c|}{$\alpha$-glucosidase inhibitors } \\
\hline Yes & $0(0.0)$ & $3(100.0)$ & \multirow[t]{2}{*}{$P=0.079^{c}$} \\
\hline No & $125(57.6)$ & $92(42.4)$ & \\
\hline \multicolumn{4}{|l|}{ GLP-I agonists } \\
\hline Yes & $\mathrm{I}(100.0)$ & $0(0.0)$ & \multirow[t]{2}{*}{$P=1.000^{c}$} \\
\hline No & $124(56.6)$ & $95(43.4)$ & \\
\hline \multicolumn{4}{|c|}{ DPP-4 inhibitors } \\
\hline Yes & $7(58.3)$ & $5(4 \mid .7)$ & \multirow[t]{2}{*}{$P=1.000^{c}$} \\
\hline No & $118(56.7)$ & $90(43.3)$ & \\
\hline \multicolumn{4}{|c|}{ Insulin (in combination) } \\
\hline Yes & $35(39.8)$ & $53(60.2)$ & \multirow[t]{2}{*}{$P<0.00 I^{b, *}$} \\
\hline No & $90(68.2)$ & $42(31.8)$ & \\
\hline Biguanides mon & rapy & & \\
\hline Yes & $19(55.9)$ & I5 (44.I) & $P=1.000^{\mathrm{b}}$ \\
\hline No & $106(57.0)$ & $80(43.0)$ & \\
\hline Sulfonylureas m & therapy & & \\
\hline Yes & $40(28.4)$ & $10(21.6)$ & $P<0.00 I^{\mathrm{b}, *}$ \\
\hline No & $85(50.0)$ & $85(50.0)$ & \\
\hline Insulin monoth & & & \\
\hline Yes & $27(46.6)$ & 31 (53.4) & $P=0.092^{b}$ \\
\hline No & $98(60.5)$ & $64(39.5)$ & \\
\hline Biguanides and & nylureas & & \\
\hline Yes & $25(62.5)$ & $15(37.5)$ & $P=0.532^{b}$ \\
\hline No & $100(55.6)$ & $80(44.4)$ & \\
\hline Biguanides and & & & \\
\hline Yes & $6(28.6)$ & I5 (7I.4) & $P=0.012^{b, *}$ \\
\hline No & $119(59.8)$ & $80(40.2)$ & \\
\hline Biguanides, sulf & ureas, and DP & nhibitors & \\
\hline Yes & $2(40.0)$ & $3(60.0)$ & $P=0.654^{c}$ \\
\hline No & $123(57.2)$ & $92(42.8)$ & \\
\hline Sulfonylureas ar & sulin & & \\
\hline Yes & I (50.0) & I (50.0) & $P=1.000^{c}$ \\
\hline No & $124(56.9)$ & $94(43.1)$ & \\
\hline Biguanides, sulf & ureas, and ins & & \\
\hline Yes & $0(0.0)$ & $2(100.0)$ & $P=0.185^{c}$ \\
\hline No & $125(57.3)$ & $93(42.7)$ & \\
\hline Biguanides, sulf & ureas, DPP-4 & itors, and ir & \\
\hline Yes & I $(100.0)$ & $0(0.0)$ & $P=1.000^{c}$ \\
\hline No & $124(56.6)$ & $95(43.4)$ & \\
\hline DPP-4 inhibitor & d insulin & & \\
\hline Yes & I (I00.0) & $0(0.0)$ & $P=1.000^{c}$ \\
\hline No & $124(56.6)$ & $95(43.4)$ & \\
\hline
\end{tabular}


Table 6 (Continued)

\begin{tabular}{|c|c|c|c|}
\hline \multirow{2}{*}{$\begin{array}{l}\text { Antidiabetic } \\
\text { regimens }\end{array}$} & \multicolumn{2}{|c|}{$A_{I C}$ level $(n, \%)$} & \multirow[t]{2}{*}{$P$-value ${ }^{a}$} \\
\hline & $<\mathbf{8 . 0} \%$ & $\geq \mathbf{8 . 0} \%$ & \\
\hline \multicolumn{4}{|c|}{ Biguanides and DPP-4 inhibitors } \\
\hline Yes & I (50.0) & I (50.0) & $P=1.000^{c}$ \\
\hline No & $124(56.9)$ & $94(43.1)$ & \\
\hline \multicolumn{4}{|c|}{ Biguanides, sulfonylureas, and $\alpha$-glucosidase inhibitors } \\
\hline Yes & $0(0.0)$ & $2(100.0)$ & $P=0.185^{c}$ \\
\hline No & $125(57.3)$ & $93(42.7)$ & \\
\hline \multicolumn{4}{|c|}{ Sulfonylureas and meglitinides } \\
\hline Yes & $2(100.0)$ & $0(0.0)$ & $P=0.507^{c}$ \\
\hline No & $123(56.4)$ & $95(43.6)$ & \\
\hline \multicolumn{4}{|c|}{ Sulfonylureas and TZDs } \\
\hline Yes & I (50.0) & I (50.0) & $P=1.000^{c}$ \\
\hline No & $124(56.9)$ & $94(43.1)$ & \\
\hline \multicolumn{4}{|c|}{ Sulfonylureas and DPP-4 inhibitors } \\
\hline Yes & $3(57.1)$ & $3(42.9)$ & $P=1.000^{c}$ \\
\hline No & $|2|(56.8)$ & $92(43.2)$ & \\
\hline
\end{tabular}

Notes: ${ }^{a}$ Degree of freedom $=1$; ${ }^{\mathrm{b}} \mathrm{Pearson}$ chi-square test with continuity correction; 'Fisher exact test; *statistically significant $(P<0.05)$.

Abbreviations: $A_{I C}$, glycated hemoglobin; GLP-I agonists, glucose-like peptide-I agonists; DPP-4 inhibitors, dipeptidyl peptidase-4 inhibitors; TZDs, thiazolidinediones.

glyburide. Overall, sulfonylureas were associated with good glycemic control, which may help to improve CV profiles in T2DM patients.

Insulin

Insulin and a combination of insulin and biguanides (metformin) were associated with glycemic control. Insulin was found to be associated with poor glycemic control

Table 7 Association between CV complications and glycemic control $\left(A_{1 C}\right.$ level)

\begin{tabular}{|c|c|c|c|}
\hline \multirow[t]{2}{*}{ CVDs } & \multicolumn{2}{|c|}{$A_{I C}$ level $(n, \%)$} & \multirow[t]{2}{*}{$P$-value ${ }^{a}$} \\
\hline & $<8.0 \%$ & $\geq \mathbf{8 . 0} \%$ & \\
\hline \multicolumn{4}{|l|}{ IHD } \\
\hline Yes & 93 (97.7) & 79 (45.9) & $P=0.164^{b}$ \\
\hline No & $32(66.7)$ & $16(33.3)$ & \\
\hline \multicolumn{4}{|l|}{ ACS } \\
\hline Yes & $66(57.4)$ & $49(42.6)$ & $P=0.892^{b}$ \\
\hline No & $59(56.2)$ & $46(43.8)$ & \\
\hline \multicolumn{4}{|l|}{$\mathrm{HF}$} \\
\hline Yes & $17(58.6)$ & $12(4 \mid .4)$ & $P=0.449^{c}$ \\
\hline No & $106(56.1)$ & $83(43.9)$ & \\
\hline \multicolumn{4}{|l|}{$\mathrm{AF}$} \\
\hline Yes & $10(83.3)$ & $2(16.7)$ & $P=0.073^{c}$ \\
\hline No & $115(55.3)$ & $93(44.7)$ & \\
\hline \multicolumn{4}{|l|}{ Stroke } \\
\hline Yes & $34(69.4)$ & $15(30.6)$ & $P=0.044^{\mathrm{b}, *}$ \\
\hline No & 91 (53.2) & $80(46.8)$ & \\
\hline
\end{tabular}

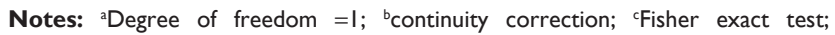
*statistically significant $(P<0.05)$.

Abbreviations: CV, cardiovascular; CVDs, cardiovascular diseases; IHD, ischemic heart disease; $\mathrm{ACS}$, acute coronary syndrome; $\mathrm{HF}$, heart failure; $\mathrm{AF}$, atrial fibrillation; $A_{I C}$, glycated hemoglobin.
Table 8 Correlation between laboratory parameters and glycemic control

\begin{tabular}{lc}
\hline Laboratory parameters & $P$-value \\
\hline LVEF & 0.155 \\
CK & 0.248 \\
CKMB & 0.275 \\
Troponin-I & 0.084 \\
TG & 0.188 \\
LDL & $0.02 I^{*}$ \\
HDL & 0.835 \\
TC & 0.107 \\
SBP & 0.773 \\
DBP & 0.352 \\
FBG & $<0.00$ I* \\
\hline Note: *Computed using Pearson Correlation and statistically significant $(P<0.05)$. \\
Abbreviations: LVEF, left ventricular ejection fraction; CK, creatinine kinase; \\
CKMB, creatinine kinase-MB; TG, triglyceride; LDL, low-density lipoprotein; HDL, \\
high-density lipoprotein; TC, total cholesterol; SBP, systolic blood pressure; DBP, \\
diastolic blood pressure; FBG, fasting blood glucose.
\end{tabular}

Table 9 Association between concurrent medications and glycemic control

\begin{tabular}{|c|c|c|c|}
\hline \multirow{2}{*}{$\begin{array}{l}\text { Concurrent } \\
\text { medications }\end{array}$} & \multicolumn{2}{|c|}{$A_{I C}$ level $(n, \%)$} & \multirow[t]{2}{*}{$P$-value } \\
\hline & $<\mathbf{8 . 0} \%$ & $\geq 8.0 \%$ & \\
\hline \multicolumn{4}{|l|}{ ACEI } \\
\hline Yes & $51(54.8)$ & $42(45.2)$ & \multirow[t]{2}{*}{$P=0.712^{b}$} \\
\hline No & $74(58.3)$ & $53(4 I .7)$ & \\
\hline \multicolumn{4}{|l|}{ ARB } \\
\hline Yes & $16(50.0)$ & $16(50.0)$ & \multirow[t]{2}{*}{$P=0.516^{b}$} \\
\hline No & $109(58.0)$ & $79(42.0)$ & \\
\hline \multicolumn{4}{|l|}{$\beta$-blocker } \\
\hline Yes & $64(6 \mid .5)$ & $40(38.5)$ & \multirow[t]{2}{*}{$P=0.229^{b}$} \\
\hline No & $61(52.6)$ & $55(47.4)$ & \\
\hline \multicolumn{4}{|l|}{$\mathrm{CCB}$} \\
\hline Yes & $61(59.2)$ & $42(40.8)$ & \multirow[t]{2}{*}{$P=0.590^{\circ}$} \\
\hline No & $64(54.7)$ & $53(45.3)$ & \\
\hline \multicolumn{4}{|l|}{ Diuretics } \\
\hline Yes & $45(52.9)$ & $40(47.1)$ & \multirow[t]{2}{*}{$P=0.462^{b}$} \\
\hline No & $79(59.0)$ & $55(4 I .0)$ & \\
\hline \multicolumn{4}{|l|}{ Nitrate } \\
\hline Yes & $31(55.4)$ & $25(44.6)$ & \multirow[t]{2}{*}{$P=0.92 \mathrm{I}^{\mathrm{b}}$} \\
\hline No & $94(57.3)$ & $70(42.7)$ & \\
\hline \multicolumn{4}{|l|}{ Fibrate } \\
\hline Yes & $8(47.1)$ & $9(52.9)$ & \multirow[t]{2}{*}{$P=0.398^{b}$} \\
\hline No & 117 (58.2) & $85(42.3)$ & \\
\hline \multicolumn{4}{|l|}{ Statin } \\
\hline Yes & $100(57.1)$ & 75 (42.9) & \multirow[t]{2}{*}{$P=0.982^{b}$} \\
\hline No & $25(55.6)$ & $20(44.4)$ & \\
\hline \multicolumn{4}{|c|}{$\alpha$-receptor blocker } \\
\hline Yes & II (6I.I) & 7 (38.9) & \multirow[t]{2}{*}{$P=0.892^{b}$} \\
\hline No & $114(56.4)$ & $88(43.6)$ & \\
\hline \multicolumn{4}{|l|}{ Anticoagulants } \\
\hline Yes & $8(57.1)$ & $6(42.9)$ & \multirow[t]{2}{*}{$P=1.000^{b}$} \\
\hline No & $117(56.8)$ & $89(44.6)$ & \\
\hline \multicolumn{4}{|l|}{ Antiplatelets } \\
\hline Yes & $103(55.4)$ & $12(35.3)$ & \multirow[t]{2}{*}{$P=0.4 \mathrm{I}^{\mathrm{b}}$} \\
\hline No & $22(64.7)$ & $12(35.3)$ & \\
\hline
\end{tabular}

(Continued) 
Table 9 (Continued)

\begin{tabular}{|c|c|c|c|}
\hline \multirow{2}{*}{$\begin{array}{l}\text { Concurrent } \\
\text { medications }\end{array}$} & \multicolumn{2}{|c|}{$A_{I C}$ level $(n, \%)$} & \multirow[t]{2}{*}{$P$-value ${ }^{a}$} \\
\hline & $<8.0 \%$ & $\geq 8.0 \%$ & \\
\hline \multicolumn{4}{|c|}{ Hematinic agents } \\
\hline Yes & $25(6 \mid .4)$ & 14 (35.9) & \multirow[t]{2}{*}{$P=0.404^{b}$} \\
\hline No & $100(55.2)$ & $81(44.8)$ & \\
\hline \multicolumn{4}{|l|}{ Supplements } \\
\hline Yes & $21(63.6)$ & $12(36.4)$ & \multirow[t]{2}{*}{$P=0.505^{b}$} \\
\hline No & $104(55.6)$ & $83(44.4)$ & \\
\hline \multicolumn{4}{|c|}{ Gout preparations } \\
\hline Yes & 7 (87.5) & I (I2.5) & \multirow[t]{2}{*}{$P=0.142^{c}$} \\
\hline No & $118(55.7)$ & $94(44.3)$ & \\
\hline \multicolumn{4}{|l|}{$\mathrm{H} 2 \mathrm{RA}$} \\
\hline Yes & I5 (7I.4) & $6(28.6)$ & \multirow[t]{2}{*}{$P=0.242^{b}$} \\
\hline No & $110(55.6)$ & $89(44.9)$ & \\
\hline \multicolumn{4}{|l|}{ PPI } \\
\hline Yes & $34(66.7)$ & $17(33.3)$ & \multirow[t]{2}{*}{$P=0.145^{b}$} \\
\hline No & 91 (53.8) & $78(46.2)$ & \\
\hline \multicolumn{4}{|c|}{ Antidepressants } \\
\hline Yes & $3(50.0 \%)$ & $3(50.0)$ & \multirow[t]{2}{*}{$P=1.000^{\circ}$} \\
\hline No & $|2|(56.8)$ & $92(43.2)$ & \\
\hline
\end{tabular}

Notes: a Degree of freedom $=\mathrm{l}$; ${ }^{\mathrm{P}}$ Pearson chi-square test with continuity correction; 'Fisher exact test.

Abbreviations: $A C E I$, angiotensin converting enzyme inhibitor; $A R B$, angiotensin receptor blocker; CCB, calcium channel blocker; H2RA, histamine 2 receptor antagonist; PPI, proton pump inhibitor; $\mathrm{A}_{I C}$, glycated hemoglobin.

Table 10 Association between comorbidities and glycemic control

\begin{tabular}{|c|c|c|c|}
\hline \multirow[t]{2}{*}{ Comorbidities } & \multicolumn{2}{|c|}{$A_{I C}$ level $(n, \%)$} & \multirow[t]{2}{*}{$P$-value ${ }^{a}$} \\
\hline & $<8.0 \%$ & $\geq 8.0 \%$ & \\
\hline \multicolumn{4}{|l|}{ Renal disease } \\
\hline Yes & $59(62.1)$ & $36(37.9)$ & $P=0.214$ \\
\hline No & $66(52.8)$ & $59(47.2)$ & \\
\hline \multicolumn{4}{|l|}{ Hepatic disease } \\
\hline Yes & $3(30.0)$ & $7(70.0)$ & $P=0.105^{b}$ \\
\hline No & $22(58.1)$ & $88(41.9)$ & \\
\hline \multicolumn{4}{|l|}{ Retinopathy } \\
\hline Yes & $13(50.0)$ & $13(50.0)$ & $P=0.592^{b}$ \\
\hline No & $112(57.7)$ & $82(42.3)$ & \\
\hline \multicolumn{4}{|l|}{$\mathrm{BPH}$} \\
\hline Yes & $10(90.9)$ & I (9.I) & $P=0.026^{\mathrm{b}, *}$ \\
\hline No & II $5(55.0)$ & $94(45.0)$ & \\
\hline \multicolumn{4}{|l|}{ Hyperlipidemia } \\
\hline Yes & $52(57.8)$ & $38(42.2)$ & $P=0.920^{c}$ \\
\hline No & $73(56.2)$ & $57(43.8)$ & \\
\hline \multicolumn{4}{|l|}{ Obesity } \\
\hline Yes & $22(55.0)$ & $18(45.0)$ & $P=0.86 \mathrm{I}^{\mathrm{c}}$ \\
\hline No & $103(57.2)$ & 77 (42.8) & \\
\hline \multicolumn{4}{|l|}{ Pneumonia } \\
\hline Yes & $5(100.0)$ & $0(0.0)$ & $P=0.07 I^{b}$ \\
\hline No & $120(55.8)$ & $95(44.2)$ & \\
\hline \multicolumn{4}{|l|}{ Anemia } \\
\hline Yes & $8(72.7)$ & $3(27.3)$ & $P=0.358^{b}$ \\
\hline No & $117(56.0)$ & $92(44.0)$ & \\
\hline \multicolumn{4}{|l|}{ Hypertension } \\
\hline Yes & $98(59.0)$ & $68(41.0)$ & $P=0.314^{c}$ \\
\hline No & $27(50.0)$ & $27(50.0)$ & \\
\hline
\end{tabular}

Notes: ${ }^{\mathrm{D} D e g r e e}$ of freedom $=\mathrm{I}$; ${ }^{\mathrm{b}}$ Fisher exact test; ${ }^{\mathrm{C}}$ Pearson chi-square test with continuity correction; *statistically significant $(P<0.05)$.

Abbreviations: $\mathrm{A}_{I C}$, glycated hemoglobin; $\mathrm{BPH}$, benign prostatic hyperplasia.
Table II Association between demographic and clinical characteristics and glycemic control

\begin{tabular}{|c|c|c|c|}
\hline \multirow{2}{*}{$\begin{array}{l}\text { Clinical and demographic } \\
\text { characteristics }\end{array}$} & \multicolumn{2}{|c|}{$A_{I C}$ level $(n, \%)$} & \multirow[t]{2}{*}{$P$-value } \\
\hline & $<\mathbf{8 . 0} \%$ & $\geq \mathbf{8 . 0} \%$ & \\
\hline \multicolumn{4}{|l|}{ Sex } \\
\hline Male & $66(55.5)$ & $53(44.5)$ & \multirow[t]{2}{*}{$P=0.76 I^{a}$} \\
\hline Female & $59(58.4)$ & $42(4 \mid .6)$ & \\
\hline \multicolumn{4}{|l|}{ Age } \\
\hline Nonelderly & $54(48.6)$ & 57 (5I.4) & \multirow[t]{2}{*}{$P=0.018^{\mathrm{a}, *}$} \\
\hline Elderly & $70(65.4)$ & $37(34.6)$ & \\
\hline \multicolumn{4}{|l|}{ Ethnicity } \\
\hline Malay & $45(54.9)$ & $37(45.1)$ & \multirow[t]{4}{*}{$P=0.344^{b}$} \\
\hline Chinese & $36(65.5)$ & $19(34.5)$ & \\
\hline Indian & $43(52.4)$ & $39(47.6)$ & \\
\hline Other & I (I00.0) & $0(0.0)$ & \\
\hline \multicolumn{4}{|l|}{$\mathrm{BMI}$} \\
\hline Underweight $(<18.5)$ & I (50.0) & I (50.0) & \multirow[t]{5}{*}{$P=0.958^{b}$} \\
\hline Normal weight (18.5-22.9) & $15(60.0)$ & $10(40.0)$ & \\
\hline Preobese (23.0-27.4) & $19(55.9)$ & $15(44.1)$ & \\
\hline Obese $(\geq 27.5)$ & $22(52.4)$ & $20(47.6)$ & \\
\hline Unknown & $68(58.1)$ & $49(4 \mid .9)$ & \\
\hline \multicolumn{4}{|l|}{ Smoking status } \\
\hline Yes & $12(48.0)$ & $13(52.0)$ & \multirow[t]{4}{*}{$P=0.207^{b}$} \\
\hline No & $80(55.2)$ & $65(44.8)$ & \\
\hline Ex-smoker & $23(60.5)$ & $15(39.5)$ & \\
\hline Unknown & $10(83.3)$ & $2(16.7)$ & \\
\hline \multicolumn{4}{|l|}{ Duration of T2DM (years) } \\
\hline$<1$ & I (33.3) & $2(66.7)$ & \multirow[t]{7}{*}{$P=0.185^{b}$} \\
\hline $1-5$ & $10(58.8)$ & $7(4 \mid .2)$ & \\
\hline $6-10$ & $27(75.0)$ & $9(25.0)$ & \\
\hline $11-15$ & $15(5 \mid .7)$ & $14(48.3)$ & \\
\hline $16-20$ & $9(50.0)$ & $9(50.0)$ & \\
\hline$>20$ & $25(47.2)$ & $28(52.8)$ & \\
\hline Unknown & $38(59.4)$ & $26(40.6)$ & \\
\hline
\end{tabular}

Notes: ${ }^{\text {PPearson }}$ chi-square test with continuity correction; ${ }^{\text {}}$ Fisher exact test; *statistically significant $(P<0.05)$.

Abbreviation: $A_{1 C}$, glycated hemoglobin; T2DM, type 2 diabetes mellitus; BMI, body mass index.

in this study. This is consistent with Adham et $\mathrm{al}^{16}$ who showed that patients treated with insulin or a combination of insulin and hypoglycemic agents had poor glycemic control. This may be because patients treated with insulin or a combination of insulin and other oral antidiabetic agents had more severe comorbid illnesses and uncontrolled diabetes, and thus required more aggressive treatment to control their illnesses. ${ }^{16}$

In addition, hypoglycemic effect, weight gain, and the anabolic effect of insulin also contributed to the poor glycemic control associated with insulin. ${ }^{16}$ Insulin is associated with an increased risk of hypoglycemic effects. A study by Parsaik et a ${ }^{17}$ showed that insulin contributed to the highest percentage of hypoglycemia cases requiring ambulance services. Weight gain is another common problem related to the use of insulin because of counteracting hypoglycemic 
complications and changing adiposity regulators. ${ }^{18}$ However, a study by Meneghini et a ${ }^{19}$ revealed that insulin detemir improved glycemic control with less hypoglycemic risk and no weight gain in T2DM patients. In this study, the combination of subcutaneous isophane insulin and soluble insulin was the most common type of insulin regimen received by patients. A study by Choi et $\mathrm{al}^{20}$ revealed that long-term continuous subcutaneous insulin infusion therapy in T2DM patients was associated with optimal glycemic control and an improvement in $\beta$-cell function.

\section{Association between cardiovascular complications and glycemic control}

In this study, stroke was the only CV complication found to be associated with glycemic control; no significant association was found between glycemic control and IHD, ACS, HF, and AF. Studies by Kauffman et al ${ }^{18}$ and Penno et $\mathrm{al}^{6}$ showed that glycemic control was not associated with CV complications. This finding was in contrast to the United Kingdom Prospective Diabetes Study (UKPDS) in which intensive glycemic control had a significantly positive effect on patients with CVD after a 10-year follow-up. ${ }^{21}$ The Heart Outcome Prevention Evaluation (HOPE) study also reported a contradictory finding, in which the glycemic level was significantly associated with incident $\mathrm{CV}$ complications. ${ }^{22}$ This difference could be because of the small sample size ( $n=220$ patients) recruited in this study and the short duration of follow-up compared with the UKPDS trial and HOPE study. Thus, additional research is needed to determine a definitive association between glycemic control and CVDs.

\section{Stroke}

Stroke was found to be significantly associated with good glycemic control in this study. This finding was in contrast to the study conducted by Ray et $\mathrm{al}^{23}$ which reported that glycemic control (targeted FPG less than $6 \mathrm{mmol} / \mathrm{L}$ ) had no significant effect on stroke or all-cause mortality. A systemic review conducted by Bellolio et $\mathrm{al}^{24}$ concluded that glycemic control by intravenous insulin administration in acute ischemic stroke did not significantly affect functional outcome, final neurological deficit, or death. The discrepancy may be due to some potential biases in the review process in the study by Bellolio et $\mathrm{al}^{24}$ which may cause inaccurate interpretation of results. For example, a moderate number of studies (eleven trials) were included in the analysis, and there were a small number of subjects in each study. ${ }^{24}$ A study by Baird et $\mathrm{al}^{25}$ revealed that infarcts expanded more in diabetic patients than in nondiabetic patients, which indicates that hyperglycemia was associated with a change in infarct volume. However, there was no evidence showing that a reversal in hyperglycemia could improve the prognosis in stroke. ${ }^{26}$ Because the association between glycemic control and stroke is still not clearly elucidated, further investigation is needed.

\section{Association between laboratory parameters and glycemic control LDL cholesterol}

This study found a significant, positive correlation between $\mathrm{A}_{1 \mathrm{C}}$ and elevated LDL cholesterol. This result is consistent with a study conducted by Adham et $\mathrm{al}^{16}$ which revealed that LDL cholesterol was significantly associated with glycemic control. Dyslipidemia is a common comorbidity in T2DM patients with $\mathrm{CV}$ complications, and LDL cholesterol is the therapeutic target for dyslipidemia treatment, which may affect glycemic control. ${ }^{27}$ However, our results did not clarify whether lipid control and glycemic control were related because the majority of patients were already on lipid-lowering regimens, such as statins and fibrates. Some studies also showed that statins can affect glycemic control in T2DM patients. ${ }^{28,29}$ For example, a study by Nakata et $\mathrm{al}^{28}$ showed that atorvastatin treatment significantly increased $\mathrm{A}_{1 \mathrm{C}}$ but not FPG in diabetic patients. This was because it impaired glucose metabolism on account of insulin resistance and decreased SLC2A4 expression in white adipose tissue. This showed that atorvastatin could potentially affect glycemic control. Thus, the elevated LDL cholesterol level often adds an additional burden to patient conditions and complicates their therapies.

\section{Association between comorbidities and glycemic control}

\section{Benign prostatic hyperplasia}

There was a significant association between BPH and glycemic control in this study. There was a higher percentage of males compared with females in this study, which may explain the significant association between BPH and glycemic control. Elderly patients with $\mathrm{BPH}$ have a higher prevalence of CVDs than the general population, ${ }^{30}$ because BPH may be related to higher blood pressure, IHD, or other CV pathologic conditions. However, the underlying mechanisms are unclear, and further studies are needed to identify whether BPH is related to CVD. ${ }^{30}$ A study by Sarma et $\mathrm{al}^{31}$ demonstrated that there was no significant association between glycemic control and BPH characteristics such as 
prostate growth and prostate-specific antigen level in T2DM patients with BPH. A study by Shionoiri et $\mathrm{al}^{32}$ showed that doxazosin, which is a BPH medication, improved glucose intolerance by significantly reducing $\mathrm{A}_{1 \mathrm{C}}$. In addition, a study by Derosa et $\mathrm{al}^{33}$ showed the synergistic effects of doxazosin and acarbose, an $\alpha$-glucosidase inhibitor that improves glycemic control, in patients with impaired glucose tolerance. Further research is needed to determine the relationship between BPH and glycemic control.

\section{Association between concurrent medications and glycemic control Statins}

In this study, statins were not significantly associated with glycemic control. This was consistent with Zhou et $\mathrm{al}^{34}$ who reported that statin therapy did not significantly affect glycemic control in patients with T2DM. However, the same study showed that atorvastatin had a detrimental effect on $A_{1 C}$ in subgroup analysis. A study by Simsek et $\mathrm{al}^{35}$ also reported the same results, ie, that high-dose atorvastatin (atorvastatin $80 \mathrm{mg}$ ) reduced glycemic control. In this study, less than $10 \%$ of patients received atorvastatin, which could be why the result was not significant. The majority of these patients $(65.5 \%)$ received simvastatin because it had a better effect on $\mathrm{A}_{1 \mathrm{C}}$ compared with atorvastatin. ${ }^{34}$ Thus, statin therapy did not affect glycemic control in this study.

\section{Antiplatelet therapy}

No significant association was found between antiplatelet therapy and glycemic control. Approximately $45 \%$ of patients were taking aspirin, and $23 \%$ were taking aspirin together with clopidogrel. A study by Singh et $\mathrm{al}^{36}$ reported that different doses of aspirin had no significant effect on inflammation, oxidative stress, insulin resistance, or endothelial function in T2DM. Thus, aspirin, which has a cardioprotective effect, did not affect glycemic control in T2DM.

\section{Diuretics}

Diuretics were not significantly associated with glycemic control. This contrasts with a study by Blackburn and Wilson, ${ }^{37}$ who showed that thiazide diuretics were associated with adverse effects on glycemic control because they decreased insulin sensitivity, resulting in increased hepatic glucose production. However, this effect was less apparent with a lower dose of thiazide (12.5-25 mg of hydrochlorothiazide) in this study, and, therefore, the result was not significant. In the diuretic-treated group, the majority of patients received furosemide (61.2\%), and its effect on glycemic control was not significant. Thus, there was no significant association between diuretics and glycemic control.

\section{Association between demographic and clinical characteristics with glycemic control \\ Age}

In this study, elderly patients were associated with good glycemic control, and nonelderly patients were associated with poor glycemic control. This finding was consistent with several studies, which reported that a younger age was associated with worse glycemic control. ${ }^{16,38}$ In this study, there was a higher percentage of nonelderly patients than elderly patients. The younger group of patients may be less motivated to manage their diabetic condition, because they may be busy with their job and have less time to comply with a healthy lifestyle, medication, and clinic visits. Effective education and behavior medication programs should be aimed at the younger population because this group of patients may have a longer life expectancy, and it is important to prevent complications associated with diabetes. ${ }^{38}$

\section{Duration of diabetes}

The duration of diabetes in this study was not associated with glycemic control. In contrast to this finding, Ji et al ${ }^{39}$ reported that glycemic control was related to the duration of T2DM. This finding was consistent with Chan et $\mathrm{al}^{40}$ who showed that glycemic control was positively correlated with diabetes duration. When duration of T2DM increased, the number of oral antidiabetic agents also increased, and insulin therapy intensified. ${ }^{41}$

\section{Study strength}

This study has several strengths. First, in this cross-sectional and retrospective study, all factors such as antidiabetic agents and glycemic control (exposure), CV profile (outcome) and comorbidities and concurrent medications (confounders) were measured. Data were also collected on the whole study population at a single time to examine the association between antidiabetic agents and glycemic control with CV complications. Specific types of CV complications were included such as IHD, ACS, AF, HF, and stroke, which represent most of the Malaysian population. In this study, the duration of antidiabetic agents was at least 3 months, and exposure to these agents was well defined. In addition, $A_{1 C}$ values for each patient were collected and recorded, which made the association between glycemic control and $\mathrm{CV}$ complications more reliable. 


\section{Study limitations}

The retrospective nature of the study design caused the whole data collection process to be based solely on information available from patients' medical records. Dependence on medical records tends to introduce bias and produce inaccurate results, especially in circumstances where there are missing data on patients' information that is relevant to the study. Important information might also be neglected or deliberately omitted due to illegible handwriting. Patients' adherence to antidiabetic medications also cannot be assessed unless adherence status is recorded in the case notes.

\section{Conclusion}

Sulfonylureas in combination, insulin in combination, sulfonylurea monotherapy, and insulin and metformin combination therapy were associated with glycemic control in this population. Overall, the antidiabetic regimen was strongly correlated with glycemic control. Stroke was the only type of $\mathrm{CV}$ complication found to be associated with glycemic control. BPH was the only comorbidity that appeared to be significantly associated with glycemic control. No concurrent medication was found to be significantly associated with glycemic control. Other factors like LDL cholesterol level and age were correlated with glycemic control. The identification of underlying factors associated with glycemic control may help in preventing $\mathrm{CV}$ progression in T2DM patients. Thus, additional care is required for patients with multiple risk factors.

\section{Acknowledgment}

The authors thank the University of Malaya, Malaysia (RP024B-14HTM), for financial and technical support.

\section{Disclosure}

The authors report no conflicts of interest in this work.

\section{References}

1. Hu FB. Globalization of diabetes: the role of diet, lifestyle, and genes. Diabetes Care. 2011;34(6):1249-1257.

2. International Diabetes Federation. Diabetes Atlas. 5th ed. Brussels, Belgium: IDF; 2011

3. Wan Nazaimoon WM, Md Isa SH, Wan Mohamad WB, et al. Prevalence of diabetes in Malaysia and usefulness of $\mathrm{HbA} 1 \mathrm{C}$ as a diagnostic criterion. Diabet Med. 2013;30(7):825-828.

4. American Diabetes Association. Standards of Medical Care in Diabetes. Diabetes Care. 2014;36:S14-S87.

5. World Heart Federation. Cardiovascular Disease Risk Factors: Diabetes. Geneva, Switzerland: World Heart Federation; 2014. Available from: http://www.world-heart-federation.org/cardiovascular-health/ cardiovascular-disease-risk-factors/diabetes/. Accessed March 26, 2014.
6. Penno G, Solini A, Zoppini G, et al. Hemoglobin A1C variability as an independent correlate of cardiovascular disease in patients with type 2 diabetes: a cross-sectional analysis of the renal insufficiency and cardiovascular events (RIACE) Italian multicenter study. Cardiovasc Diabetol. 2013;12:98.

7. Dipiro JT, Talbert RL, Yee GC, et al. Pharmacotherapy: A Pathophysiologic Approach. 8th ed. New York, NY: McGraw-Hill Medical; 2011.

8. Thrainsdottir I, Malmberg K, Olsson A, Gutniak M, Ryden L. Initial experience with GLP-1 treatment on metabolic control and myocardial function in patients with type 2 diabetes mellitus and heart failure. Diab Vasc Dis Res. 2004;1(1):40-43.

9. Nikolaidis LA, Mankad S, Sokos GG, Miske G, Shah A. Effects of glucagon-like peptide-1 in patients with acute myocardial infarction and left ventricular dysfunction after successful reperfusion. Circulation. 2004;109(8):962-965.

10. Sokos GG, Nikolaidis LA, Mankad S, Elahi D, Shannon RP. Glucagonlike peptide-1 infusion improves left ventricular ejection fraction and functional status in patients with chronic heart failure. J Cardiac Fail. 2006;12(9):694-699.

11. Fowler MJ. Diabetes treatment part 2: oral agents for glycemic management. Clin Diabetes. 2007;25(4):131-134.

12. Shah DD, Fonarow GC, Horwich TB. Metformin therapy and outcomes in patients with advanced systolic heart failure and diabetes. $J$ Cardiac Fail. 2010;16(3):200-206.

13. Soraya H, Khorrami A, Garjani A, Maleki-Dizaji N, Garjani A. Acute treatment with metformin improves cardiac function following isoproterenol induced infarction in rats. Pharmacol Rep. 2012; 64:1476-1484.

14. Vilar L, Canadas V, Arruda MJ, et al. Comparison of metformin, gliclazide MR and rosiglitazone in monotherapy and in combination for type 2 diabetes. Arq Bras Endocrinol Metabol. 2010;54(3):311-318.

15. Schernthaner G, Grimaldi A, Di Mario U, et al. GUIDE study: doubleblind comparison of once-daily gliclazide MR and glimepiride in type 2 diabetic patients. Eur J Clin Invest. 2004;34(8):535-542.

16. Adham M, Froelicher ES, Batieha A, Ajlouni K. Glycaemic control and its associated factors in type 2 diabetic patients in Amman, Jordan. East Mediterr Health J. 2010;16(7):732-739.

17. Parsaik AK, Carter RE, Myers LA, Basu A, Kudva YC. Hypoglycemia requiring ambulance services in patients with type 2 diabetes is associated with increased long term mortality. Endocr Pract. 2013;19(1):29-35.

18. Kauffman AB, Delate T, Olson KL, et al. Relationship between haemoglobin A1C values and recurrent cardiac events. Clin Drug Investig. 2008;28(8):501-507.

19. Meneghini LF, Rosenberg KH, Koenen C, Merilainen MJ, Luddeke HJ. Insulin detemir improves glycaemic control with less hypoglycaemia and no weight gain in patients with type 2 diabetes who were insulin naive or treated with NPH or insulin glargine: clinical practice experience from a German subgroup of the PREDICTIVE study. Diabetes Obes Metab. 2007;9(3):418-427.

20. Choi SB, Lee JH, Kim S, et al. Improvement of $\beta$-cell function after achievement of optimal glycaemic control via long-term continuous subcutaneous insulin infusion therapy in non-newly diagnosed type 2 diabetic patients with suboptimal glycaemic control. Diabetes Metab Res Rev. 2013;29(6):473-482.

21. UK Prospective Diabetes Study (UKPDS) Group. Effect of intensive blood-glucose control with metformin on complications in overweight patients with type 2 diabetes (UKPDS 34). Lancet. 1998;352(9131):854-865.

22. Gerstein HC, Pogue J, Mann JF, et al. The relationship between dysglycaemia and cardiovascular and renal risk in diabetic and non-diabetic participants in the HOPE study: a prospective epidemiological analysis. Diabetologia. 2005;48(9):1749-1755.

23. Ray KK, Seshasai SR, Wijesuriya S, et al. Effect of intensive control of glucose on cardiovascular outcomes and death in patients with diabetes mellitus: a meta-analysis of randomised controlled trials. Lancet. 2009;373(9677):1765-1772. 
24. Bellolio MF, Gilmore RM, Stead LG. Insulin for glycaemic control in acute ischaemic stroke. Cochrane Database Syst Rev. 2011; 9:CD005346.

25. Baird TA, Parsons MW, Phanh T, et al. Persistent poststroke hyperglycemia is independently associated with infarct expansion and worse clinical outcome. Stroke. 2003;34:2208-2214.

26. Lindsberg PJ, Roine RO. Hyperglycemia in acute stroke. Stroke. 2004;35(2):363-364

27. Reiner Z, Catapano AL, De Backer G, et al. ESC/EAS Guidelines for the management of dyslipidaemias: the task force for the management of dyslipidaemias of the European Society of Cardiology (ESC) and the European Atherosclerosis Society (EAS). Eur Heart J. 2011;32(14):1769-1818.

28. Nakata M, Nagasaka S, Kusaka I, et al. Effects of statins on the adipocyte maturation and expression of glucose transporter 4 (SLC2A4): implications in glycaemic control. Diabetologia. 2006;49(8):1881-1892.

29. Sukhija R, Prayaga S, Marashdeh M, et al. Effect of statins on fasting plasma glucose in diabetic and nondiabetic patients. J Investig Med. 2009;57(3):495-499.

30. Karatas OF, Bayrak O, Cimentepe E, Unal D. An insidious risk factor for cardiovascular disease: benign prostatic hyperplasia. Int J Cardiol. 2010;144(3):452.

31. Sarma AV, St Sauver JL, Hollingsworth JM, et al. Diabetes treatment and progression of benign prostatic hyperplasia in community-dwelling black and white men. Urology. 2012;79(1):102-108.

32. Shionoiri H, Ashino K, Yamanaka K, et al. Effect of doxazosin therapy on glucose tolerance and lipid metabolism in hypertensive patients with impaired glucose tolerance. Clin Ther. 1997;19(3):527-536.

33. Derosa G, Cicero AF, D'Angelo A, et al. Synergistic effect of doxazosin and acarbose in improving metabolic control in patients with impaired glucose tolerance. Clin Drug Investig. 2006;26(9):529-539.

34. Zhou Y, Yuan Y, Cai RR, et al. Statin therapy on glycaemic control in type 2 diabetes: a meta-analysis. Expert Opin Pharmacother. 2013; 14(12):1575-1584.
35. Simsek S, Schalkwijk CG, Wolffenbuttel BH. Effects of rosuvastatin and atorvastatin on glycaemic control in type 2 diabetes - the CORALL study. Diabet Med. 2012;29(5):628-631.

36. Singh K, Raghavan VA. Insulin Resistance and Atherosclerosis in Lipoproteins in Diabetes Mellitus. New York, NY: Springer; 2014: 41-44.

37. Blackburn DF, Wilson TW. Antihypertensive medications and blood sugar: theories and implications. Can J Cardiol. 2006;22(3): 229-233.

38. Quah JH, Liu YP, Luo N, How CH, Tay EG. Younger adult type 2 diabetic patients have poorer glycaemic control: a cross-sectional study in a primary care setting in Singapore. BMC Endocr Disord. 2013;13(1):18

39. Ji LN, Lu JM, Guo XH, et al. Glycemic control among patients in China with type 2 diabetes mellitus receiving oral drugs or injectables. $B M C$ Pub Health. 2013;13(1):602.

40. Chan WB, Chan JC, Chow CC, et al. Glycaemic control in type 2 diabetes: the impact of body weight, $\beta$-cell function and patient education. Oxford J Med. 2000;93(3):183-190.

41. Huri HZ, Selamat S, Vethakkan SR. Factors associated with utilization of dipeptidyl-4 inhibitors in patients with type 2 diabetes mellitus: a crosssectional retrospective study. Int J Endocrinol. 2014;2014:367564. Available from: http://dx.doi.org/10.1155/2014/367564. Accessed July 16, 2015.

42. Barber N, Parsons J, Clifford S, Darracott R, Horne R. Patients' problems with new medication for chronic conditions. Quality and Safety in Health Care. 2004;13(3):172-175

43. Ministry of Health. (2004). Clinical practice guidelines on management of obesity.
Drug Design, Development and Therapy

\section{Publish your work in this journal}

Drug Design, Development and Therapy is an international, peerreviewed open-access journal that spans the spectrum of drug design and development through to clinical applications. Clinical outcomes, patient safety, and programs for the development and effective, safe, and sustained use of medicines are a feature of the journal, which

\section{Dovepress}

has also been accepted for indexing on PubMed Central. The manuscript management system is completely online and includes a very quick and fair peer-review system, which is all easy to use. Visit http://www.dovepress.com/testimonials.php to read real quotes from published authors.

Submit your manuscript here: http://www.dovepress.com/drug-design-development-and-therapy-journal 